\title{
CONFIGURACIONES DEL TRABAJO DE CUIDADOS EN EL ENTORNO FAMILIAR. DE LA TOMA DE DECISIÓN A LA GESTIÓN DEL CUIDADO
}

\section{Care arrangements within the family environment. From decision making to managing care}

ANTÍA PÉREZ CARAMÉS*

\begin{abstract}
Resumen
En este artículo trataremos la cuestión de la organización del trabajo de cuidados a mayores dependientes, analizando tanto las variables que influyen en las diferentes configuraciones de cuidados resultantes y su interrelación, como el carácter diacrónico de la toma de decisión y la articulación del trabajo de cuidado, centrando el estudio en el caso de Galicia. El objetivo es estudiar la variabilidad de articulaciones de cuidado registradas en la realidad gallega, proponiendo una tipología y arrojando pautas de explicación de los factores que entran en juego. Se aplicará una perspectiva de género en el análisis del discurso emitido por distintos miembros de las familias a la hora de tomar decisiones y organizar el trabajo de cuidados a sus familiares faltos de autonomía, entendiéndose las configuraciones de cuidados derivadas de estos procesos como articulaciones estratégicas, dinámicas y flexibles, atravesadas por relaciones de poder y lógicas de género que operan en las familias. La metodología empleada en este estudio forma parte del material recolectado por la autora para su tesis doctoral y consiste en el análisis de datos secundarios de carácter sociodemográfico y sobre políticas de atención a la dependencia, así como en entrevistas en profundidad semiestructuradas.
\end{abstract}

Palabras clave: configuraciones de cuidado, mayores dependientes, envejecimiento, género, estrategias familiares.

\footnotetext{
* Antía Pérez Caramés

Universidad de A Coruña

Facultad de Sociología

Campus de Elviña, s/n. A 15071 La Coruña

Teléfono: 981167000 (extensión: 4851) Fax: 981167103

antia.perez@udc.es
} 


\begin{abstract}
This paper considers the issue of how care for elderly people is organised, analysing the variables that influence the various ways in which care is configured and how they interrelate, as well as the diachronic nature of decision making and how care work is co-ordinated in Galicia. The aim is to study how variable the co-ordination of care is in Galicia, and to describe and explain the factors involved. A gender perspective is applied in the analysis of the discourse by various family members when making decisions and organising care for relatives lacking in autonomy, in which the configurations of care resulting from these processes are seen as strategic, dynamic and flexible forms of co-ordination combined with the power relationships and gender differences that operate in families. The methodology used in this study forms part of the material gathered by the author for her doctoral thesis and consists of an analysis of secondary socio-demographic data and dependent care policies, as well as in-depth semi-structured interviews.
\end{abstract}

Keywords: organisation of care, care-dependent elderly persons, ageing, gender, family strategies.

\title{
1. Introducción y contextualización: la «crisis de cuidados» y el pertinaz familismo en los Estados de Bienestar del sur de Europa
}

La reciente intensificación sobre el debate en relación a las políticas de atención a la dependencia en España viene enmarcada en un contexto que ha sido bautizado como la «crisis de los cuidados» (Pérez de Orozco, 2006), expresión que resume la convergencia de varios procesos sociales y demográficos que desembocan en una menor disponibilidad de cuidadores informales para hacer frente a una creciente demanda de asistencia. Por una parte, y dentro del espectro demográfico, el creciente aumento de la población mayor de 65 años, así como de su proporción sobre el conjunto de la población, han colocado a España en la órbita de los países más envejecidos de Europa. Por otra parte, el cambio en las expectativas personales y profesionales de las mujeres ha conllevado a una progresivamente mayor incorporación de éstas al trabajo formal y una menor disponibilidad para las ocupaciones vinculadas con las necesidades de reproducción social, fenómeno relacionado también con una transición tranquila en la tipología predominante de estructuras familiares en España (Jurado, 2005) que ha debilitado las redes sociales con las que contaban los mayores.

A continuación, analizaremos, a continuación, cuáles han sido la intensidad y magnitud de estos procesos en los últimos quince años a la luz de diversa información estadística. 
Gráfico 1. Evolución del peso de la tercera edad y de la esperanza de vida al nacer para hombres y mujeres en el periodo 1991-2005

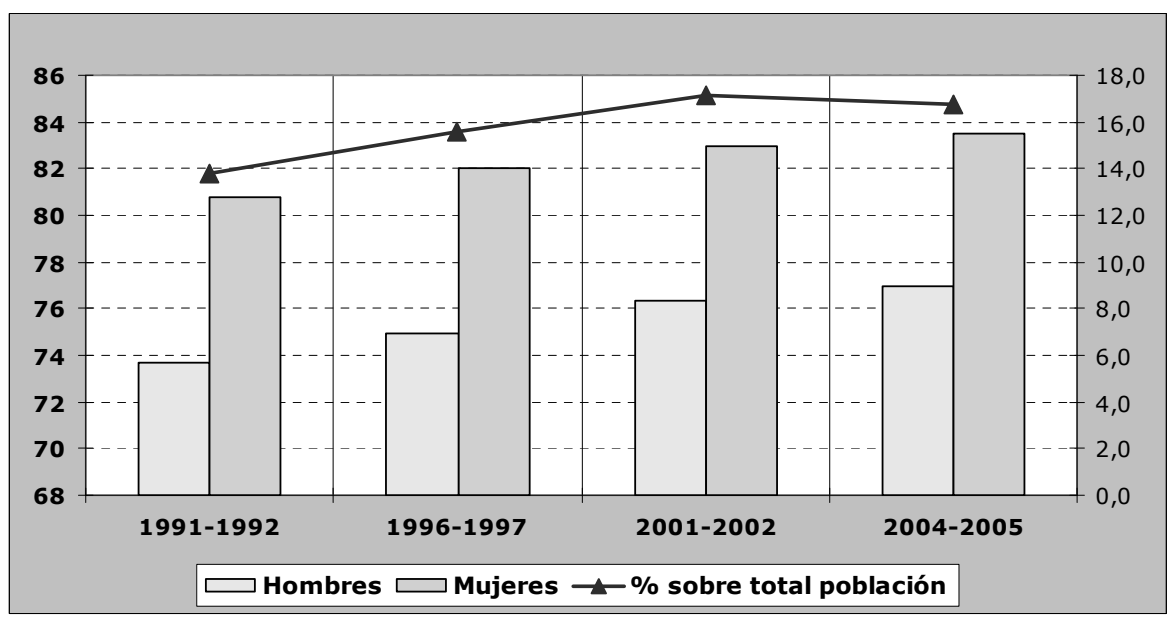

Fuente: Padrón Municipal de Habitantes, años 1996, 2001 y 2006. Censo de Población y Viviendas, año 2001. Tablas de Mortalidad, años 1991-1992, 1996-1997, 2001-2002 y 2004-2005. Instituto Nacional de Estadística (INE), <http://www.ine.es>.

Nota: la esperanza de vida se expresa en años en el eje de la izquierda y el peso de la tercera edad en años en el eje de la derecha.

En el Gráfico 1 se observa de manera comparada la evolución del indicador relativo al peso de la tercera edad ${ }^{1}$ en España, así como de la esperanza de vida al nacer de hombres y mujeres durante el periodo que media entre 1991-2006. En una franja temporal de sólo quince años, el peso de los mayores sobre el conjunto de la población española ha aumentado en tres puntos porcentuales, pasando de más de cinco millones de personas con más de 65 años a prácticamente siete millones y medio ${ }^{2}$. Paralelamente, la esperanza de vida al nacer, tanto de hombres como de mujeres, ha aumentado, en ambos casos, en casi tres años.

1. Este indicador ha sido calculado dividiendo la población total de 65 años o más entre el total de la población de España a una fecha dada y expresando el resultado en términos porcentuales.

2. Según los datos del Censo de Población y Viviendas del INE para 1991, el número de personas de edad igual o superior a los 65 años de edad era de 5370252 , mientras que los datos del Padrón Municipal de Habitantes (INE) a principios de enero de 2006 arrojan una cifra de 7484392 personas de 65 años o más. 
Gráfico 2. Tasas de actividad de las mujeres españolas por grupos de edad entre 1999-2005

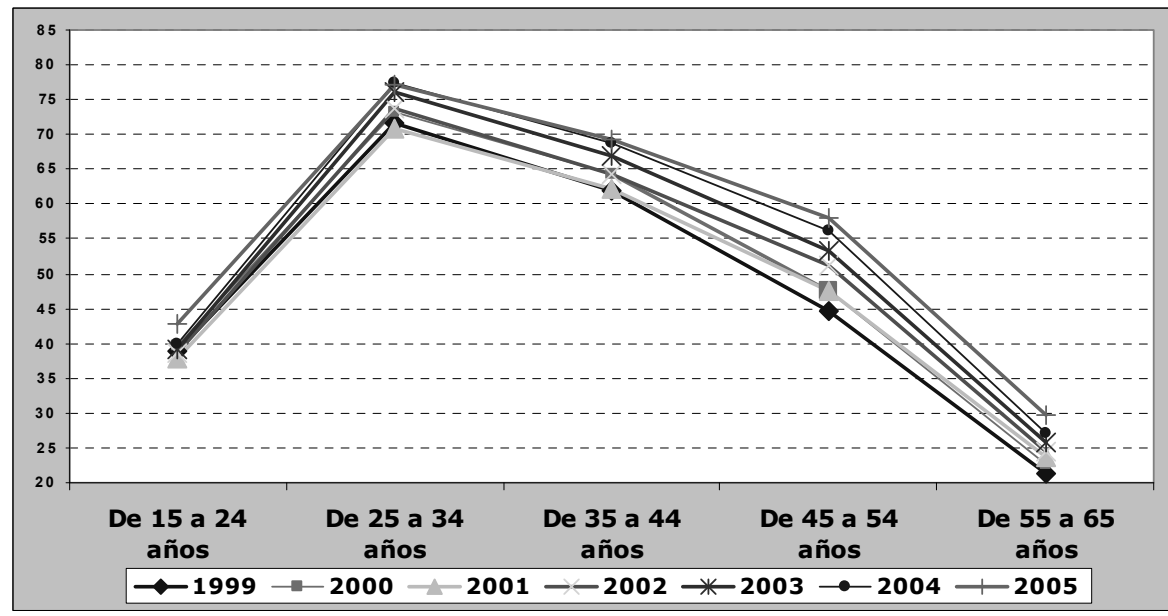

Fuente: Encuesta de Fuerza de Trabajo de la UE (EU Labour Force Survey) 1999-2005. Instituto Nacional de Estadística (INE).

En segundo lugar, nos concentraremos en el análisis de la evolución de las tasas de actividad de las mujeres en España en función de su edad (ordenada en grandes grupos) en un periodo de tiempo reciente, entre 1999-2006. A la luz del Gráfico 2 podemos comprobar cómo las tasas de participación en el mercado laboral de las mujeres en nuestro país han crecido en todos los grupos de edad considerados, pudiendo constatarse, además, que el mayor crecimiento en términos porcentuales lo experimentan las mujeres situadas entre los 45 y los 54 años de edad, que registran una participación en el mercado laboral del 58,1 \% en el 2005, frente al 44,6 \% de 1999; esto es, un aumento de más de trece puntos porcentuales. En suma, vemos, pues, que las nuevas generaciones de mujeres están aumentando notablemente la participación laboral de sus predecesoras a todas las edades, especialmente en aquellas de mayor edad. La disponibilidad de cuidadoras informales familiares es, en consecuencia, claramente decreciente, por lo que es urgente la necesidad de optar por modelos políticos de atención a la dependencia que resulten eficientes para evitar y/o aminorar las consecuencias que el desempeño del cuidado tiene para el trabajo de las mujeres (Sarasa, 2008), y que no contemplen la díada trabajo-cuidados como una relación de suma cero (Bussemaker, 1998).

Si bien es cierto que el fenómeno de la «crisis de los cuidados» ha desatado las alarmas en buena parte de los países industrializados, no deja de 
ser menos evidente que el contexto de bienestar en que se registren estos fenómenos influirá en buena medida en la capacidad de unos y otros estados para hacer frente a esta crisis. Para proporcionar el marco general, así como las principales características del Estado de Bienestar español en relación con la protección de los mayores dependientes, realizaremos un repaso teórico a las principales contribuciones a este respecto.

La publicación de la obra de Esping-Andersen en la que se hace un recorrido por los diferentes sistemas de bienestar, relacionándolos con los niveles de desigualdad social, para concluir con la proposición de una tipología de Estados de Bienestar (Esping-Andersen, 1993), ha desencadenado ríos de tinta en dos vertientes diferenciadas pero concluyentes en su trayectoria. Por una parte, los estudiosos del Estado de Bienestar de los países del sur de Europa centraron su crítica en la existencia de un conglomerado de países, agrupado bajo el calificativo de "conservadores», que no respondían a las mismas características en sus esquemas de protección social. Si bien compartían el hecho de ser sistemas que basaban los derechos de ciudadanía en el elemento contributivo, países como España, Italia, Portugal y Grecia poseían como rasgo diferenciador respecto de Estados como Francia o Alemania el papel preeminente otorgado a la familia en el desempeño de la protección social (Sarasa y Moreno, 1995; Rhodes, 1997; Moreno, 2006). Este carácter familista de los Estados de Bienestar del sur de Europa se correlacionaba, además, con otros indicadores como la baja participación femenina en el trabajo remunerado -con la única excepción de Portugal-, así como con unas tasas de fecundidad extremadamente reducidas (Flaquer, 2004). A juicio de estos investigadores, la tipología de Esping-Andersen debía ser remozada e incluir una nueva categoría, la de los Estados de Bienestar mediterráneos o los Estados de Bienestar del sur de Europa, para dar cuenta de la heterogeneidad existente dentro del grupo de los Estados de Bienestar conservadores.

Por otra parte, un buen aluvión de críticas llovieron al esquema propuesto por Esping-Andersen por parte de las investigadoras en materia de género y políticas públicas (Lewis, 1998). Nancy Fraser critica el concepto de desfamiliarización empleado por Esping-Andersen, al aducir que no necesariamente se deben concebir las políticas relacionadas con el cuidado y la conciliación de la vida laboral y familiar con las posibilidades de remercantilización de las mujeres, ya que se puede también incitar a los hombres a realizar el «trabajo invisible de las mujeres» (Fraser, 1996). Por otra parte, Diane Sainsbury indica que la variable género atraviesa y fragmenta los diferentes regímenes del Estado de Bienestar, siendo necesario evaluar la relación dinámica existente entre las lógicas políticas de los regímenes de género y los regímenes del 
Estado de Bienestar. Las políticas y la distribución del trabajo de cuidado a familiares dependientes se convierten, dentro de esta perspectiva, en un elemento fundamental para evaluar la estructura de las desigualdades de género en los diferentes regímenes de bienestar (Sainsbury, 1999).

Así, se ha señalado que los regímenes de bienestar mediterráneos comportan el máximo de desigualdad de género, al considerar primordialmente a las mujeres, en función de sus roles familiares, como reproductoras y cuidadoras y desprotegerlas del mercado en caso de necesidad económica al no promover la reconciliación entre los roles familiares y los laborales ${ }^{3}$ (Trifiletti, 1999).

El familismo, en tanto que instrumento conceptual para caracterizar a los Estados de Bienestar de la Europa Meridional, se vuelve un arma de doble filo al invisibilizar la estructura completamente desigualitaria de las relaciones de género en los regímenes de bienestar del sur de Europa, ya que afirmar que es la familia sobre quien recae el peso de la protección social supone hacer tabla rasa de la profunda inequidad existente en la distribución de los roles familiares entre hombres y mujeres en estos países (Leitner, 2003). S. Leitner, tras realizar un análisis de las políticas de apoyo a las familias en las funciones de cuidado, llega a la conclusión de que las políticas de los regímenes de bienestar de la Europa del sur -a las que atribuye un familismo explícito generizado- se caracterizan, no sólo por mantener a la familia dentro de su función cuidadora, sino que además refuerzan el rol cuidador de las mujeres, perpetuando, de este modo, la división generizada del cuidado familiar.

En su clasificación de regímenes de cuidado, las autoras F. Bettio y J. Plantenga, partiendo de indicadores tales como el tiempo que se espera sea dedicado al cuidado (de niños y/o mayores dependientes), la provisión de servicios disponibles para el cuidado y la inactividad laboral vinculada al desempeño de trabajos de cuidado, concluyen con la afirmación de que países como España delegan toda la gestión del cuidado a la familia (Bettio y Plantenga, 2004).

Otras autoras han vinculado la caracterización de los regímenes de bienestar con la expansión de la atención informal familiar y el trabajo informal de las inmigrantes en los sectores del servicio doméstico a hogares y de cuidado de niños y mayores dependientes (Woods, 2007; Kofman, 2006). Así, Eleanore Kofman, empleando el concepto de cuidado social (social care) que postulan las investigadoras Mary Daly y Jane Lewis (Daly y Lewis, 2000), caracteriza a los regímenes migratorios y de bienestar de la Europa del sur como aquellos que proveen escasos servicios de cuidado (tanto para niños como para mayores), que cuentan con una reducida tasa de participación laboral de

3. Como sí promueven, en cambio, los modelos de bienestar basados en el hombre ganapán. 
las mujeres nativas y que emplean abundante mano de obra inmigrante en el servicio doméstico a hogares y no, en cambio, en los servicios sociales.

España se encuentra, pues, en la peor de las situaciones posibles para enfrentarse a la "crisis de los cuidados». Y es que si la recientemente aprobada Ley de Promoción de la Autonomía Personal y Atención a las personas en situación de dependencia ha recibido el calificativo de «cuarto pilar» del Estado de Bienestar en España, desde luego las familias y, más concretamente, las mujeres en el seno de las familias, deberían consecuente y justamente ser apodadas como el «cimiento» del bienestar español.

\section{El apoyo formal e informal a los mayores en situación de dependencia en España: una interacción contraproductiva para las mujeres}

Según diversos trabajos del investigador Gregorio Rodríguez Cabrero, podríamos estimar que en España contamos con una población mayor en situación de dependencia de entre 1583234 y 1444750 personas (Rodríguez Cabrero, $2005 a, b$ y 2007$)^{4}$. Si tomamos los datos relativos al conjunto de la población española a comienzos del 2005 que nos proporciona el Padrón Municipal de Habitantes ${ }^{5}$, nos encontramos con que los mayores dependientes representan entre un 3,6 \% y un 3,3\% del total de la población.

Por otra parte, el investigador Rodríguez Cabrero apunta, además, que el modelo de protección social a la dependencia existente en España se define como un modelo dual, con un componente mayoritariamente informal, basado en la centralidad del trabajo no remunerado de las mujeres y su consecuente disponibilidad para prestar cuidados informales y subsidiariamente asistencial (Rodríguez Cabrero, 2005a). Este modelo aparece, por ende, refrendado por la Ley 9/1998, de 15 de julio, que hace a las familias responsables del cuidado de sus mayores ${ }^{6}$, responsabilidad familiar fijada por ley que España comparte con otros países de la órbita del bienestar mediterráneo (Naldini, 2002).

4. De entre las distintas estimaciones que el profesor Rodríguez Cabrero recoge en sus obras, hemos escogido aquella basada en la Encuesta sobre Discapacidades, Deficiencias y Estado de Salud (EDDES) -realizada en el 2005 por el Instituto Nacional de Estadística- por ser una cifra reciente (año 2005) y por estar estimada empleando los indicadores relativos a las Actividades Básicas de la Vida Diaria (ABVD) y a las Actividades Instrumentales de la Vida Diaria (AIVD), frecuentemente utilizados en Gerontología para la evaluación de la dependencia funcional de los individuos.

5. Hemos utilizado la población a esta fecha, ya que el número estimado de mayores dependientes es para el 2005. La población de España a 01-01-2005 era de 44108560 personas según el Padrón Municipal de Habitantes (INE).

6. Se trata de la Ley 9/1998, de 15 de julio, del Código de Familia. 
Tabla 1. Comparativa de los índices de cobertura de distintos servicios de atención a la dependencia en 1999 y 2008

\begin{tabular}{|c|c|c|c|c|}
\hline AÑO & SAD & Teleasistencia & $\begin{array}{c}\text { Centros de } \\
\text { día }\end{array}$ & $\begin{array}{c}\text { Residencias (plazas } \\
\text { públicas privadas y } \\
\text { concertadas }\end{array}$ \\
\hline 1999 & 1,67 & 0,72 & 0,11 & 2,95 \\
\hline 2008 & 4,69 & 4,72 & 0,83 & 4,44 \\
\hline
\end{tabular}

Fuente: Informe 2006 y 2008 sobre las Personas Mayores en España.

IMSERSO, <http://www.seg.social.es/imserso/>.

Así, si analizamos los índices de cobertura de los diferentes servicios sociales destinados a las personas mayores con algún grado de dependencia, nos encontramos con que, pese a la progresión experimentada desde 1999, la capacidad de asumir públicamente la gestión del cuidado a los mayores dependientes resulta todavía claramente deficiente.

De entre aquellos servicios con mayor cobertura, destacan precisamente los residenciales, herencia del tardofranquismo en que la pauta predominante en la atención a los mayores faltos de autonomía se basaba en el principio de la institucionalización. Sin embargo, desde comienzos de los años ochenta, el paradigma de la desinstitucionalización y la defensa del cuidado comunitario entra con fuerza en los discursos de la política social española (Bazo, 1998). El primer Plan Gerontológico Nacional, aprobado en 1992, hace suyo el lema de «envejecer en casa» y busca promover toda una serie de iniciativas conducentes a promover la permanencia del mayor en su entorno el máximo tiempo posible. Se argumenta que esta fórmula es menos costosa humana y económicamente, pero esto es únicamente desde la perspectiva del mayor dependiente, y no de su cuidadora informal en el seno de la familia (Escuredo, 2007). Esta política de mantenimiento en el domicilio del mayor no ha ido aparejada, sin embargo, de un aumento sustancial del gasto social en los años siguientes a la aprobación de este plan, por lo que las mujeres han tenido que hacer frente a una sobrecarga de trabajo en un contexto, tal y como hemos indicado, de aumento de la participación laboral femenina, escasez de oferta de empleo a tiempo parcial y cicatería de recursos sociales para el cuidado de los familiares en situación de dependencia (Guillén, 1997).

Por otra parte, los cuidadores informales han sido considerados como recursos (según la tipología de cuidadores informales formulada por Twigg, 1990) al servicio del Estado para garantizar la atención a los mayores dependientes, ya que las medidas de ayuda al cuidador (tales como el denominado 
«respiro familiar» a través de estancias temporales en residencias y centros de día) y los permisos laborales por razones de atención a un familiar dependiente desarrollados a través de la Ley de Conciliación de la Vida Laboral y Familiar ${ }^{7}$ son claramente insuficientes (Escuredo, 2007) y, en ocasiones, desincentivan la participación laboral de las mujeres (Pérez de Orozco y Baeza Gómez, 2006).

\section{Objetivos y metodología}

En primer lugar y, de modo general, el presente trabajo se propone identificar, describir y analizar, a partir de contextos concretos de investigación, el proceso de construcción social a través del que emerge la figura de la cuidadora informal en el seno de las familias, y cómo ésta se configura en "gestora del cuidado» de su pariente dependiente. En este proceso de emergencia de la cuidadora informal familiar, se hará especial hincapié en el análisis de los procesos de toma de decisión realizados en el seno de las familias ante la entrada en situación de dependencia de uno de sus miembros, o el agravamiento de esta situación. Por lo tanto, el segundo de los objetivos definidos en este estudio es determinar la influencia de la socialización diferencial por género de los miembros de la familia, y cómo esta asunción de roles diferenciados genera lógicas de género que moldean las actitudes, opiniones y estrategias de los distintos miembros frente a la necesidad de asistencia a un familiar dependiente, creando, de este modo, una normatividad refrendada en el entorno social y familiar más próximo y apoyada en la socialización diferencial por género. Partimos de la premisa de que los juicios sobre «lo que es correcto hacer» son variados y complejos y muchas personas no responden al mismo patrón de obligaciones filiales (Finch y Mason, 1993). Las investigaciones de Simon Duncan y Rosalind Edwards, así como la obra de Janet Finch, han resaltado el papel de las normas sociales y las lógicas de género a la hora de moldear las actitudes de los actores sociales en la toma de decisiones relativas al cuidado, como un patrón normativo incorporado socialmente que se utiliza para dar sentido a los comportamientos (Duncan y Edwards, 1999; Finch, 1987).

Por último, se evalúa el papel de distintos factores estructurales en las estrategias y lógicas de género derivadas de los procesos decisionales ante las necesidades asistenciales de un miembro de la familia. Entre estas variables, hemos considerado, por su importancia contrastada en otros estudios sobre los determinantes del cuidado informal en el seno de las familias, el tipo y

7. Hablamos de la Ley 39/1999, de 5 de noviembre, de Conciliación de la Vida Laboral y Familiar. 
tamaño del hogar en el que reside el mayor dependiente, la posición relativa de las mujeres en el mercado de trabajo en el contexto más amplio del hogar, la relación de parentesco del cuidador con la persona asistida, así como las posibilidades económicas del hogar de cara a evaluar las posibilidades de externalización del trabajo de cuidado. Así, los estudios de Claudine AttiasDonfut han puesto de relieve las diferencias en cuanto a la percepción de las obligaciones sociales en función de la clase social de pertenencia (AttiasDonfut, 1995). Sobre la influencia de los hijos en los procesos de toma de decisión relativa a los cuidados, afirmando que estas decisiones no pertenecen ni se gestan en exclusiva por parte de las personas mayores carentes de autonomía, resultan de especial interés para nuestro trabajo las aportaciones de Clara Pratt y sus colegas, así como las de T. Brubaker (Pratty y otros, 1989; Brubaker, 1987; Pratt, Jones y Pennington, 1993). En torno al factor de corresidencia en relación a las decisiones relativas a la provisión de cuidados, Liliana Pezzin, R. A. Pollak y B. Schone han mostrado la reducción de la capacidad de negociación en caso de corresidencia de los hijos con los mayores dependientes (Pezzin, Pollak y Schone, 2005).

En cuanto a la metodología, su carácter es cualitativo. El trabajo de campo ha consistido en la realización de 20 entrevistas en profundidad a mujeres responsables del cuidado de un familiar en situación de dependencia, así como a otros miembros del entorno familiar del mayor dependiente -principalmente hijos varones de la persona necesitada de cuidados. En lo referido a la observación participante, ésta se ha realizado en el seno de dos familias que contaban con una persona mayor que no podía valerse por sí misma.

\section{La toma de decisión ante situaciones de dependencia: roles, estrategias y lógicas de género}

Dado que uno de los objetivos principales ha sido analizar cómo se gestan los procesos de decisión relativa a la provisión de cuidados que necesita un familiar dependiente, la primera de las constataciones derivadas del análisis del trabajo de campo realizado es que parece desprenderse a primera vista la inexistencia de un proceso o momento en el que se toma el acuerdo acerca de quién y cómo provee el cuidado al familiar necesitado, pareciera tratarse de un mecanismo de toma de decisión que no implica la reunión voluntaria de la familia (Engers y Stern, 1998; Stern, 1995; Hiedemann y Stern, 1999). Muchas de las entrevistadas llegaban incluso a aludir al destino para explicar por qué ellas, y no otro familiar, se hicieron cargo en su momento de la asistencia al mayor: 
«Fue como un castigo divino. Ya cuando era mayor yo decía: “¿A que me va a tocar a mí esto de cuidar de mi madre?" " (Hija cuidadora de su madre con Alzheimer).

Tirando del hilo de la aparente fatalidad, enseguida constatamos que la toma de decisión acerca de la gestión familiar del cuidado no puede cifrarse en un momento concreto del tiempo, ni siquiera puede circunscribirse al hecho aislado de la entrada en situación de dependencia de un miembro de la familia; se trata, más bien, de un proceso decisional subrepticio y subyacente, que en no pocas ocasiones se retrotrae hasta la etapa de socialización adolescente de la cuidadora, que, a menudo, no es consciente de los pactos intergeneracionales que lleva implícita una determinada decisión en un momento dado.

«Ella, no sé, quizás por su carácter, porque pasó muchos trabajos con los niños (yo estaba todo el día fuera de casa) se involucró mucho, de lleno con los críos. (...) Ahora, cualquier cosa que le hagas ya te dice: "Lo tienes que hacer porque yo esto ya lo hice por tus hijos". Mi madre tiene la actitud de "Mira, yo lo hice por ti, fastídiate, que ahora tú lo tienes que hacer".» (Hija cuidadora de su madre y de su padre).

Así pues, la responsabilidad del cuidado de un mayor dependiente entra dentro de un contrato de género más amplio, que, si bien en un momento facilita la inserción laboral de las mujeres de la familia -al ser los mayores los que se encargan de las tareas de cuidado de los más pequeños de la casa-, posteriormente puede ejercer el efecto contrario, el de desmercantilización obligada de las mujeres que entenderán el trabajo de cuidar de sus mayores como la reciprocidad normativa inherente al contrato intergeneracional y de género o, como lo define Constanza Tobío, la «doble lógica de consanguinidad y de género» (Tobío, 2002), implícitamente suscrito.

En hogares intergeneracionales en los que la cuidadora es hija de la persona atendida y existe corresidencia entre ambos, es frecuente además que la decisión del cuidado sea un eslabón más de un conjunto de intercambios, no solamente del trabajo de cuidado, sino también económicos entre generaciones que restringen todavía más la capacidad de la cuidadora a la hora de formular estrategias para enfrentarse a la problemática de la dependencia sin considerar el abandono del trabajo formal remunerado. La «hija de la casa», beneficiada económicamente con respecto a sus hermanos y sus otras hermanas, y que tiene, además, garantizado el cuidado de sus hijos y, consecuentemente, el poder trabajar fuera de casa ya que convive con sus padres, es perfectamente consciente de que puede llegar un momento en el que tenga que desempeñar su parte del contrato, y expresa, en estas circunstancias, un sentimiento de resignación para el que ya estaba preparada y, en ocasiones, 
abandona el trabajo remunerado porque, de todas formas, ya sabía que su situación laboral podía ser temporal.

«Sabes lo típico de las herencias, también. Mis padres eran muy burros, pero para eso fueron muy listos. Mi padre tuvo muchos problemas, y de hecho su herencia quedó sin repartir entre sus hermanos. Entonces ellos, lo que era de ellos se lo dejaron a cada hijo, pero ellos -en referencia a los hermanos de la entrevistada- piensan que me dejaron más, y sí es verdad que a mí me dejaron más, pero ellos piensan que como a mí me dejaron más, pues tengo más obligación. (...) También al estar viviendo aquí, algo vas aportando, y no vas a aportar toda la vida para que al final sea para las siete partes igual. (...) Ya cuando mi padre vivía, me metieron en la misma cuenta que ellos, para que yo pudiese igualmente que ellos hacer de su dinero.» (Hija cuidadora de su madre con Alzheimer).

«También ella cuidó de mis hijos para que yo trabajase, si ahora yo tengo que dejar de trabajar para cuidarla a ella, es lo mínimo que puedo hacer.» (Hija cuidadora de su madre con Alzheimer).

Si no estamos ante el caso de hogares intergeneracionales, la influencia normativa del contrato de género se diluye, y el espectro de estrategias posibles que pueden adoptar las mujeres responsables del cuidado familiar se amplía. Aquí, el proceso de decisión y la emergencia de la cuidadora informal familiar se gestan en la transición del care about («preocuparse por») al care for («cuidar de») (Hooyman y Gonyea, 1995).

«Al principio de morir mi padre, ella lo que sí hizo desde el primer momento fue venir a dormir a mi casa, porque como que le daba así un poco de..., sentía la soledad y esas cosas.»

(...) Yo se lo comenté a mis hermanos. Ella tenía miedo a perderse, el miedo se apoderó de ella y yo la ayudé a tranquilizarse.

(...) Hasta que no le empezó el proceso nada, que ella era completamente autónoma, pero al ir empezando este proceso, empecé a tener que irme encargando yo de las cosas. (...) Luego empezaron las movilizaciones de médicos, se pasó en el hospital como un mes y medio; ella no me lo pidió, pero yo me quedé en su casa.» (Hija cuidadora de su madre con Alzheimer).

La transición aparentemente tranquila del «preocuparse por» al «cuidar de» se rebela contra la propia cuidadora cuando ésta se vuelve consciente de que realmente ha pasado de preocuparse a trabajar intensamente en tareas de cuidado. Esta transición suele ser un momento de crisis, y en ella son partícipes otros familiares directamente relacionados con la persona asistida de la necesidad de «tomar una decisión» - por primera vez, las entrevistadas hacen referencia a esta expresión- respecto del desempeño del cuidado de su pariente. Es en este momento, y no antes, cuando el factor de clase social entra en juego para determinar y posibilitar la externalización del cuidado o no. 
«Cuando estallé con mis hermanos y les dije que yo ya no aguantaba más esa situación, empecé a relatarles la cantidad de cosas que yo tenía que hacer con mi madre; ellos no eran para nada conscientes. (...) Hice una relación de cosas que hacía para decirles por qué estaba tan cansada, porque me tenía que justificar ante ellos. Les dije que estaba destrozando mi vida, que yo no tenía vida personal, que estaba deseando que la Consellería me trasladase a Ávila porque así yo no tenía que cuidarla. (...) Lo de ellos era comodidad, como tenían su coartada, se sentían liberados en ese sentido, tampoco llegaban a darse cuenta de lo implicada que estaba yo en esa situación hasta que vieron toda la lista de cosas que tenía que hacer. (...) Les dije que había que tomar una decisión. (...)». (Hija cuidadora de su madre con Alzheimer).

La utilización de las palabras «justificar»y «coartada» por parte de la entrevistada en este extracto de su entrevista, para referirse respectivamente a la expresión de la sobrecarga del trabajo de cuidado y a la exclusión de la participación de otros miembros de la familia en el desempeño de estas tareas, nos remite inmediatamente al vocabulario jurídico. La entrevistada no puede expresar la carga del trabajo emocional más que a través de la justificación y la aportación de pruebas (elaboración de un listado de tareas realizadas); expresión que realiza ante sus hermanos y ante ella misma, en un proceso de toma de conciencia ante sí misma y ante sus hermanos del componente de trabajo que subyacía en lo que ella veía como la extensión emocional de una preocupación inicial. La coartada se resume en un pretexto justificante que, según la racionalidad de género expresada por la entrevistada, exime a sus hermanos de dispensar cuidados a su madre. Los hermanos devienen inculpables porque disponen de una coartada de género, que los sitúa frente al escenario social de la dependencia, en otro lugar y otra posición social.

Si la cuidadora es hija única, es muy probable que el sentimiento de obligación moral de cuidar por parte de otros familiares tenga contornos borrosos -salvo en algún caso, por parte de hermanos/as de la persona dependiente-, por lo que, ante la carencia de recursos económicos elevados, la prácticamente única estrategia viable de mantener su empleo actual a la vez que garantizar el cuidado de su padre/madre es la fragmentación del cuidado en pequeñas unidades de tarea a asignar a todo miembro de la red familiar y social del dependiente con que se cuente. Sería, pues, aplicar la técnica de cadena de montaje fordista al trabajo emocional; reproducción social en cadena que exige, al igual que el taylorismo, una férrea disciplina del trabajo emocional y la búsqueda del control de los tiempos y los procesos.

«Por la tarde tengo a una chica que es amiga de ella y viene, juegan a las cartas, ven la telenovela. Y viene siempre una tía mía, y entonces van las tres a pasear. O sea, que si no tuviera a esta chica y a mi tía tendría que tener a al- 
guien porque no puede estar sola todo el tiempo. (...) La ducha se la hago yo, aunque con la nieta se entiende muy bien.» (Hija cuidadora de sus padres).

Si la cuidadora tiene hermanos, la posibilidad de contar con recursos económicos es mayor; y, además, si entre los hermanos hay un varón, es probable que su sentido de la obligación filial esté más connotado con la provisión de recursos económicos para el desempeño del cuidado que con el autosacrificio (Gilligan, 1993), por lo que la estrategia de externalización es una vía muy probable, y la hija cuidadora verá salvaguardada su posición en el mercado laboral, aunque nada evita, tal y como veremos en el siguiente apartado, que, durante el proceso de toma de decisión conjuntamente con sus hermanos, ella se instituya en la máxima responsable de la supervisión del trabajo de cuidado, esto es, en la gestora del cuidado.

«Mi hermano, cuando vio aquello -en referencia a la lista de tareas-, dijo que se pagaba lo que fuese. (...) Y contratamos a una chica. (...) Empecé a encargarme yo, o sea, le pusimos una mujer por horas. La contraté y yo le dije: "Tú tienes que acompañarla a ella, no dejarla nunca sola, tienes que hacerle la comida, y en casa sola no quiero nunca que esté." (Hija cuidadora de su madre con Alzheimer).

\section{Construcción social y emergencia de las «gestoras del cuidado»}

Según Barbara Da Roit, una de las estrategias empleadas parar reducir la sobrecarga de la cuidadora informal familiar consiste en la externalización al mercado privado del trabajo de cuidado mediante la contratación de una persona, generalmente inmigrante, para la realización de las correspondientes tareas (Da Roit, 2007), estrategia que ha sido analizada y desarrollada con profusión para el caso de España por la investigadora Raquel Martínez Buján (Martínez Buján, 2005 y 2007). Sin embargo, ésta es una decisión que, tal y como hemos señalado anteriormente, no se produce siempre que existe sobrecarga de la cuidadora informal familiar, sino que existen un proceso previo que sienta las condiciones normativas para que el contrato intergeneracional y de género se diluya, así como unos requisitos materiales y económicos para poder hacer frente a la externalización del cuidado. No sólo la clase social importa, sino también la presencia de hijos varones en la familia, más dispuestos a aportar en moneda que en especies.

Para Martínez Buján, la externalización de las tareas de cuidado no agota la responsabilidad de las cuidadoras informales familiares, al devenir estas «gestoras del cuidado» o cuidadoras secundarias directas del pariente dependiente (Finley, 1989). Este proceso ha sido constatado también en nuestro trabajo de campo, y consiste en una cesión del trabajo físico con el mayor 
dependiente y una supervisión muy estricta sobre el trabajo emocional delegado que, en ocasiones, es compartido, ya que el trabajo desempeñado por la cuidadora contratada no colma las expectativas de la gestora del cuidado, tal y como se desprende del extracto de la última entrevista mencionada en el punto anterior.

Sin embargo, el papel de las gestoras del cuidado va más allá de la mera supervisión y responsabilidad constante sobre las atenciones dispensadas a su pariente. El rol de supervisión que se arrogan las gestoras del cuidado, antes cuidadoras informales familiares en régimen intensivo, resalta el potencial valor de mercado que este trabajo tiene y desemboca en lo que Nancy R. Hooyman y Judith Gonyea denominan extensión del cuidado (extended care), y que definen como la traslación del apoyo a la comunidad más cercana en situaciones semejantes de contar con un familiar falto de autonomía para la realización de las tareas de la vida diaria (Hooyman y Gonyea, 1995). Con ciertos matices, este proceso ha sido constatado en varias de las cuidadoras informales familiares entrevistadas, especialmente en aquellas que han abandonado su trabajo formal para dedicarse al trabajo de cuidado de algún familiar dependiente. Así, estas personas se convierten en algo así como «cuidadoras profesionales» para su familia y su entorno más próximo, y se muestran dispuestas a cuidar de otro familiar más en caso de que esta persona caiga en situación de dependencia, e incluso realizan trabajos de cuidado remunerado fuera del hogar para compensar la pérdida de recursos generada por la desmercantilización.

«Yo ahora mismo voy aquí, a la casa de al lado, la que está pegada, que también tienen una señora dependiente y les hago las cosas de la casa, y saco a pasear a la señora. Este señor con el que estoy trabajando me está pagando el seguro de hogar -se refiere a la cotización a la Seguridad Social a través del Régimen Especial de Empleados Domésticos-, me paga el seguro de ocho horas, sólo voy dos, pero en vez de pagar, cotiza por mí.

(...) Yo le dije a mi marido -en referencia a los suegros de ella- si quieren venir para aquí, sí que los cuido, porque una vez que cuidas a uno, también cuidas a otro.» (Hija cuidadora de su madre con Alzheimer).

En la experiencia de estas mujeres, el trabajo de cuidado da el salto de la reproducción social a la producción social, haciéndose visible como trabajo formal lo que se invisibilizaba, bajo las lógicas de género, en un deber familiar.

\section{Conclusiones}

Hemos tratado de exponer cómo se articula y arregla la asistencia informal familiar a los mayores dependientes en el seno de las familias, atendiendo a 
aspectos que se han demostrado cruciales para el entendimiento de los procesos de toma de decisión relativa al cuidado, tales como la socialización temprana en roles de género, las lógicas y normativas de género interiorizadas por nuestras informantes; $y$ hemos definido el abanico de posibilidades estratégicas en torno a las decisiones de cuidado, teniendo en cuenta la especial incidencia de variables como la composición y el tamaño del hogar, la relación de parentesco de la cuidadora informal con el mayor dependiente, la posición laboral de la cuidadora informal en el contexto inmediato del hogar y el estatus socioeconómico del hogar.

No quisiéramos finalizar este trabajo, sin hacer un breve comentario crítico acerca del cambio más reciente y de mayor calado de la política social española en relación a la atención a la dependencia, que viene dado por la aprobación, a finales del 2006, de la Ley de Promoción de la Autonomía Personal y Atención a las personas en situación de dependencia, así como de la creación del Sistema Nacional de Dependencia (SND). Esta ley parte de un concepto de dependencia doblemente restringido. En primer lugar, restringido a la dependencia permanente, por lo que deja a las puertas de la protección a todas aquellas personas que temporalmente experimenten una situación de falta de autonomía (Hernando, 2006); y, en segundo lugar, restringido a la dependencia funcional, excluyendo toda aquella necesidad de cuidados que tienen las personas a lo largo de su ciclo vital y de su vida cotidiana, siguiendo el concepto más amplio que de dependencia y cuidados plantea S. Sevenhuijsen (1998) y que quedan, nuevamente, invisibilizados (Bosch, Carrasco y Grau, 2006).

Por otra parte, se trata de una ley reguladora del derecho a recibir una prestación de dependencia de difícil encaje, tanto en el sistema de Seguridad Social como en el Sistema Nacional de Salud, por lo que el encaje de esta prestación resulta algo ambiguo (López Cumbre, 2006; Berrocal Vela, 2006). Como sugiere también Lourdes López Cumbre, así como la investigadora Bibiana Escuredo, esta ley hace más hincapié en la promoción de la autonomía que en la protección de la dependencia y, sin embargo, de entre los servicios que enumera, considera únicamente la teleasistencia como recurso para la promoción de la autonomía personal (López Cumbre, 2006; Escuredo, 2007).

En relación a los cuidadores informales de las personas dependientes, y pese al énfasis político puesto en la visibilización del cuidado informal que se lograba a través de la aprobación y puesta en marcha de las medidas contempladas por esta ley, lo cierto es que esta normativa tiene como objetivo a los dependientes, y trata a sus cuidadores informales nuevamente como recurso al servicio del desarrollo del sistema de protección a la dependencia en ella 
contemplado. No se mejoran y amplían las medidas recogidas en la Ley de Conciliación de la Vida Laboral y Familiar, ni se cuestiona la obligatoriedad de las familias a la hora de prestar atención y cuidados a sus dependientes, tal y como afirma Bibiana Escuredo. Además, la fórmula propuesta para la figura de los cuidadores informales incide nuevamente en la consideración del trabajo de cuidados como un «no-trabajo», al estar obligadas las cuidadoras informales a darse de alta y cotizar en la Seguridad Social para recibir la prestación (que no salario), sin, no obstante, contar con derechos laborales (Hernando, 2006). Por otra parte, si se tratase en el caso de la figura del cuidador familiar, tal y como se recoge en el texto de dicha ley, de una figura de carácter excepcional, no se entiende por qué se prevé que en el 2015 vaya a haber entre 300000 y 400000 cuidadoras familiares.

En conclusión, se trata de una ley que resulta continuista en cuanto al tratamiento de la problemática de la atención informal a la dependencia y que no resuelve la cuestión de la obligatoriedad legal y moral de las familias a prestar cuidados a sus familiares dependientes de una manera provechosa para las mujeres.

\section{Bibliografía}

Attias-Donfut, C., «Le Double Circuit de Transmission», en Les solidarités entre générations, C. Attias Donfut (ed.), París, Nathan, 1995.

Bazo, M. T., «Vejez dependiente, políticas y calidad de vida», Papers, n. ${ }^{\circ}$ 56, 1998 , pp. 143-161.

BerRoCal Vela, M. A., «Consideraciones críticas sobre el anteproyecto de Ley de Promoción de la Autonomía Personal y Atención a las Personas en Situación de Dependencia», Revista Jurídica de les Illes Balears, n. ${ }^{\circ}$ 4, 2006, pp. 153-169.

Bettio F. y J. Plantenga, «Comparing Care Regimes in Europe», en Feminist Economics, 10 (1), 2004, pp. 85-113.

Bosch, A., C. Carrasco y E. Grau, «La Ley de Dependencia y el Mito del Homo Economicus», en Revista de Economía Crítica, n. ${ }^{\circ}$ 5, 2006, pp. 105-107.

BRUBAKER, T., «The long-term care triad: the elderly, their families and bureaucracies», en Aging, Health and Family: Long-term Care, T. H. Brubaker (ed.), Beverly Hills, Sage, 1987, pp. 12-22.

BussemakeR, J., «Rationales of Care in Contemporary Welfare States: The Case of Childcare in the Netherlands», en Social Politics, 5 (1), 1998, pp. 70-96.

DA RoIT, B., "Changing Intergenerational Solidarities within Families in a Mediterranean Welfare State: Elderly Care in Italy», en Current Sociology, 55 (2), 2007, pp. 251-270.

DalY, M. y J. LEWIS, «The Concept of Social Care and the Análisis of Contemporary Welfare States», en British Journal of Sociology, 52 (2), 2000, pp. 281-298. 
DUNCAN, S. y R. EDWARDS, Lone mothers, paid work and gendered moral rationalities, Londres, Macmillan, 1999.

ENGERS, M. y S. STERN, «Long-Term Care and Family Bargaining», en Virginia Economics Online Papers, n. ${ }^{\circ} 320$, Universidad de Virginia, [en línea], 1998, <http://www.virginia.edu/economics/RePEc/vir/virpap/papers/virpap320. pdf>. [Consulta: 13/03/2008].

ESCUREDO, B., «Las políticas sociales de dependencia en España: contribuciones y consecuencias para los ancianos y sus cuidadores informales», en Revista Española de Investigaciones Sociológicas (REIS), n. ${ }^{\circ} 119,2007$, pp. 65-89.

EsPING-ANDERSEN, G., Los tres mundos del Estado del Bienestar, Valencia, Alfons el Magnánim, 1993.

FINCH, J., «Family obligations and the life course», en Rethinking the Life Cycle A. Bryman, P. Allatt y T. Keil (eds.), Londres, Macmillan, 1987, pp. 155-169.

FINCH, J. y J. MASON, Negotiating Family Responsibilities, Londres, Tavistock/ Routledge, 1993.

FINLEY, N. J., «Theories of Family Labor as applied to Gender Differences in Caregiving for Elderly Parents», en Journal of Marriage and the Family, 51 (1), 1989, pp. 79-86.

FLAQUER, L., «La articulación entre familia y Estado del Bienestar en los países de la Europa del Sur», en Papers, n. ${ }^{\circ}$ 73, 2004, pp. 27-58.

Fraser, N., «Gender Equity and the Welfare State: A Postindustrial Thought Experiment», en Democracy and Difference: contesting the Boundaries of the Political, S. Benhabib, Princeton, Princeton University Press, 1996, pp. 219-241

Gilligan, C., In a Different Voice. Psychological Theory and Women's Development, Cambridge:, Harvard University Press, 1993.

GuillÉn, A. M., «Regímenes de bienestar y roles familiares: un análisis del caso

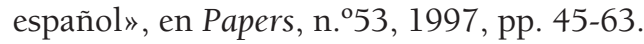

Hernando, A., «Una crítica feminista a la Ley de Dependencia», en Viento Sur, n. ${ }^{\circ} 88,2006$, pp. 89-95.

Hiedemann, B. y S. Stern, «Strategic Play Among Family Members When Making Long-Term Care Decisions», en Virginia Economics Online Papers, n. ${ }^{\circ}$ 321, Universidad de Virginia, [en línea], 1999, <http://www.virginia.edu/ economics/RePEc/vir/virpap/papers/virpap321.pdf>. [Consulta: 13/03/2008].

Hooyman, N. y J. Gonyea, Feminist Perspectives on Family Care. Policies for Gender Justice, Londres, SAGE, 1995.

IMSERSO, Informe 2006 sobre las personas mayores en España, Madrid, MTAS/ IMSERSO, 2006.

JURADO, T., «Las nuevas familias españolas», en Tres décadas de cambio social en España, J. J. González Rodríguez y M. Requena Díez de Revenga (coords.), Madrid, Alianza, 2005, pp. 51-80. 
Kofman, E., «Gendered Migrations, Social Reproduction and Welfare Regimes: New Dialogues and Directions», [en línea], 2006, <http://www2.iisg.nl/esshc/ programme9606.asp? selyear $=8 \&$ pap $=4596>$. [Consulta: $13 / 03 / 2008$ ] .

LEITNER, S., "Varities of Familialism: the Caring Function of the Family in Comparative Perspective», en European Societies, 5 (4), 2003, pp. 353-375.

LEWIS, J. , Gender, Social Care and Welfare State Restructuring in Europe, Aldershot, Ashgate, 1998.

LÓPEZ Cumbre, L., «Fisuras de una ley histórica en materia de protección social: el proyecto de ley de dependencia», en Revista General de Derecho del Trabajo y Seguridad Social, n. ${ }^{\circ}$ 11, 2006, pp. 1-17.

MARTínEZ BUJÁN, R., «El cuidado de ancianos: un vínculo entre la inmigración y el envejecimiento», en Panorama Social, n. ${ }^{\circ}$ 2, 2005, pp. 86-97.

- Bienestar y Cuidados: el Oficio del Cariño. Mujeres Inmigrantes y Mayores Nativos, tesis doctoral, Universidad de La Coruña, 2007.

MORENO, L., España y el bienestar mediterráneo, documento de trabajo 06-10 de la Unidad de Políticas Comparadas (CSIC), [en línea], 2006, <http://www. iesam.csic.es/doctrab2/dt-0610.pdf>. [Consulta: 11/03/2008].

Naldini, M., The Family in the Mediterranean Welfare States, Londres, Frank Cass, 2002.

Pérez de Orozco, A. y P. BAEZA GÓmeZ, «Sobre "dependencia” y otros cuentos. Reflexiones en torno a la Ley de Promoción de la Autonomía Personal y Atención a las Personas en Situación de Dependencia», en Revista de Relaciones Laborales / Lan Harremanak, n. ${ }^{\circ}$ 2, 2006, pp. 13-40.

PÉrez Orozco, A., «Amenaza tormenta: la crisis de los cuidados y la reorganización del sistema económico», en Revista de economía crítica, n. ${ }^{\circ}$ 5, 2006, pp. 7-37.

Pezzin, L. E., R. A. Pollak y B. Schone, «Efficiency in Family Bargaining: Living Arrangements and Caregiving Decisions of Adult Children and Disabled Elderly Parents», en NBER Working Paper, n. ${ }^{\circ}$ 12358, [en línea], 2005, <http:// www.nber.org/papers/w12358.pdf>. [Consulta: 10/03/2008].

Pratt, C., A. JoneS, y D. PenNington, «Decisión-Making Influence Strategies of Caregiving Daughters and their Elderly Mothers», en Family Relations, n. ${ }^{\circ} 42$, 1993, pp. 376-382.

PRATT, C. y otros, «Autonomy and decision-making among single women and their caregiving daughters», en The Gerontologist, n. ${ }^{\circ}$ 29, 1989, pp. 792-797.

RHodes, M., Southern European Welfare Status. Between Crisis and Reform, Londres, Routledge, 1997.

Rodríguez CABrero, G., «La protección social de las personas dependientes como desarrollo del Estado de Bienestar en España», en Panorama Social, n. ${ }^{\circ} 2$, 2005a, pp. 21-33. 
- «Conocimientos sociográficos sobre la dependencia funcional en España y su dimensión aplicada» en Avances en bienestar basados en el conocimiento, D. Casado (dir.), Madrid, CC2, 2005b, pp. 151-178.

- «Población en situación de dependencia y cuidados informales», en Temas Laborales, n. ${ }^{\circ} 89,2007$, pp. 47-62.

SAinsburY, D., Gender and Welfare State Regimes, Oxford, Oxford University Press, 1999.

SARASA, S., «Do Welfare Benefits Affect Women's Choices of Adult Care Living?», en European Sociological Review, 24 (1), 2008, pp. 37-51.

SARASA, S. y L. MORENO, (comps.), «El Estado de Bienestar en la Europa del Sur», Madrid, CSIC, 1995.

Sevenhuijsen, S., Citizenship and the Ethics of Care: Feminist Considerations on Justice, Morality and Politics, Londres, Routledge, 1998.

Stern, S., "Estimating Family Long-Term Care Decisions in the Presence of Endogenous Child Characteristics», en Journal of Human Resources, 20 (3), 1995, pp. 551-580.

ToBío, C., «Conciliación o contradicción: cómo hacen las madres trabajadoras para conciliar la vida», en Tiempos y servicios para la igualdad, C. Tobío, Madrid, Dirección General de la Mujer, 2002, pp. 15-43.

TRIFILETTI, R., «Southern European Welfare States and the Worsening Position of Women», en Journal of European Social Policy, 9 (1), 1999, pp. 49-64.

TwIGG, J., "Cuidadores de los ancianos: modelos para un análisis», en Comparación de políticas europeas de atención a las personas ancianas. A. Jamieson y R. Illsey (eds.), Madrid, SG/Fundación Caja de Madrid, 1990.

Woods, D. R., «Focusing on Care: Family Policy and Problems of Analysis», en Working Paper, $\mathrm{n}^{\circ}$ 30-2006 del Institut für Politikwissenschaft, [en línea], 2006, <http://tobias-lib.ub.uni-tuebingen.de/volltexte/2006/2309/pdf/ WIP_30.pdf>. [Consulta: 13/03/2008].

— «Shadow Worlds of the Welfare State: Examining the European Informal Domestic Sector», Gender, Work and Organization Conference, Keele 27-29 Junio, (paper). 